\title{
Tolerance of mixed lipid emulsion in neonates: effect of concentration
}

\author{
P A Cairns, D C Wilson, J Jenkins, D McMaster B G McClure
}

\begin{abstract}
Aims-To compare the effect of concentration of a mixed lipid emulsion (50:50 medium chain triglyceride/long chain triglyceride) (MCT/LCT) on lipid tolerance in neonates.

Methods-A prospective randomised controlled trial of 75 neonates requiring prolonged parenteral nutrition was conducted in the neonatal intensive care units of the Royal Maternity Hospital, Belfast, and the Waveney Hospital, Ballymena. Thirty eight infants received $10 \%$ and 37 $20 \%$ lipid emulsion. Infants were randomly assigned to groups at the start of parenteral nutrition and studied if they required seven or more days of this. Lipid tolerance was assessed by twice weekly measurements of plasma triglyceride and cholesterol concentrations and weekly measurement of non-esterified fatty acids and $\beta$ hydroxy butyrate. Anthropometry was carried out weekly.

Results-The mean cholesterol in the $10 \%$ group was significantly higher within the first seven days of the study compared with the $20 \%$ group (3.5 vs $2.87 \mathrm{mmol} / \mathrm{l}$ ), and continued to rise over the study period in contrast to the $20 \%$ group. A similar pattern was observed with the triglyceride concentrations. There was no significant difference in non-esterified fatty acids, $\beta$ hydroxy butyrate, or growth between the two groups.

Conclusion-Sick neonates show better biochemical tolerance to $20 \%$ MCT/LCT emulsion than to $10 \%$ emulsion.

(Arch Dis Child 1996;75:F113-F116)
\end{abstract}

Keywords: medium chain triglycerides, parenteral nutrition, $\beta$ hydroxy butyrate.

Royal Maternity

Hospital, Belfast

PA Cairns

BG McClure

Waveney Hospital, Ballymena

D C Wilson

J Jenkins

Department of Medicine, The Queen's

University of Belfast

D McMaster

Correspondence to: Dr PA Cairns,

Neonatal Intensive Care

Unit, Royal Maternity

Hospital, Grosvenor Road

Belfast BT12 6BB.

Accepted 24 April 1996 (enabling infusion through a peripheral vein), prevent essential fatty acid deficiency ${ }^{6}$ and permit the infusion of fat soluble vitamins. Unfortunately, premature infants have a limited, and often unpredictable, ability to utilise parenteral fat, due to low concentrations of lipoprotein lipase, ${ }^{7}$ required for the hydrolysis of triglycerides, and of carnitine, ${ }^{8}$ which is required for long chain fatty acids to enter the mitochondria where $\beta$ oxidation occurs. This may lead to lipid accumulation with impairment of neutrophil function, ${ }^{9}$ interference with macrophage activity, ${ }^{10}$ displacement of bilirubin from albumin, ${ }^{11}$ decreased oxygenation in infants with pre-existing lung disease,,$^{12}$ and possibly predisposition to bronchopulmonary dysplasia (BPD). ${ }^{13}{ }^{14}$ Medium chain triglycerides (MCT) may be more easily tolerated than long chain triglycerides (LCT) as they are metabolised independently of carnitine, have less affinity for albumin, and are more rapidly oxidised. ${ }^{15}$ In a randomised controlled trial Rubin et al demonstrated a significant lower fraction of unbound bilirubin in premature neonates given 50:50 MCT/LCT emulsion compared with those given $100 \%$ LCT emulsion. ${ }^{16}$ Lima et al, in another randomised controlled trial, reported lower plasma cholesterol concentrations in neonates given MCT/ LCT compared with those receiving LCT. ${ }^{17}$

Concentration of pure LCT emulsion is important in lipid clearance, with $20 \%$ solution being better tolerated than $10 \% .^{18}$ The aim of this study was to compare the effect of concentration on an emulsion containing equal amounts of MCT and LCT.

\section{Methods}

This study was carried out in the regional neonatal unit at the Royal Maternity Hospital, Belfast and at the Waveney Hospital, Ballymena, over an 18 month period. Infants were randomly allocated to receive either $10 \%$ or $20 \% \mathrm{MCT} / \mathrm{LCT}$ emulsion at the beginning of parenteral nutrition. Randomisation was by computer generated numbers in sealed envelopes and was stratified for birthweight. Only those infants who required seven or more days of parenteral nutrition were studied.

Parenteral nutrition was started during the first 48 hours of life when possible and followed an established protocol.$^{19}$ Both groups received an isovolumetric, isocaloric regimen, differing only in the concentration of lipid used.

Lipid infusion (Lipofundin MCT/LCT, B Braun Ltd, Melsungen, Germany) was started on the third day of life at $0.5 \mathrm{~g} / \mathrm{kg} /$ day over a 20 
hour period. This was increased daily by 0.5 $\mathrm{g} / \mathrm{kg}$ increments to a maximum of $3 \mathrm{~g} / \mathrm{kg} /$ day. In the event of hyperlipidaemia (cholesterol $>4.9 \mathrm{mmol} / 1$ or triglyceride $>1.9 \mathrm{mmol} / \mathrm{l}$ ), severe sepsis, or jaundice requiring phototherapy, the dose was decreased to $1 \mathrm{~g} / \mathrm{kg} /$ day. Parenteral nutrition was infused through a silastic percutaneous central venous catheter where possible; lipids were infused simultaneously using the same line. As enteral feeds became established, the quantity of lipid infused was reduced proportionately.

Infants left the study when full enteral feeding was established.

Anthropometry was carried out weekly. Weight was measured on electronic scales accurate to $5 \mathrm{~g}$; head circumference was measured with paper tape measure and length by neonatometer.

Blood was taken twice weekly at the end of a four hour lipid free period for cholesterol and triglyceride values. Non-esterified fatty acids and $\beta$ hydroxy butyrate concentrations were measured weekly during lipid infusion.

Cholesterol and triglyceride concentrations were analysed enzymatically using Kodak Ektachem Clinical Chemistry Slides. Nonesterified fatty acids were estimated in serum by an enzymatic colorimetric method using a kit manufactured by Wako Chemicals $\mathrm{GmbH}$, Neuss, Germany. $\beta$ hydroxy butyrate was measured using a kinetic enzymatic method with a kit supplied by Randox Laboratories Ltd, Crumlin, Northern Ireland. Initial disease severity for each infant was calculated using the CRIB score. ${ }^{20}$

Data were analysed using the Statistics for Social Sciences Package. Student's $t$ test was used for comparison of means of continuous variables. The $\chi^{2}$ test was used to compare categorical variables. A P value of less than 0.05 was regarded as significant.

Parental informed consent and approval from the Research Ethical Committee of the Queen's University of Belfast were obtained.

\section{Results}

One hundred and four infants were randomly allocated to the study but nine died (4 and 5, respectively, in the $10 \%$ and $20 \%$ groups) and 20 (10 in each group) attained full enteral feeds within seven days. Seventy five infants were therefore studied, of whom 38 were randomly allocated to $10 \%$ and 37 to $20 \%$ MCT/LCT emulsion. Two deaths occurredone in each group. Both infants died on the 10th day of life, one of pulmonary haemorrhage and one of gastric perforation. There was no significant difference between the two groups in terms of birthweight, gestational age, or requirement for ventilation in the first 24 hours (table 1). The group allocated to $20 \%$ MCT/LCT, however, was significantly sicker, having higher CRIB scores: this reflects a higher mortality risk at 12 hours.

The mean (SD) duration of lipid infusion was 15.3 (9.4) days in the $10 \%$ group and 15.6 (9.8) days in the $20 \%$ group. Figure 1 represents median parenteral lipid intake over the study period: there was no significant difference at any stage. There was no difference between the two groups in terms of daily protein, carbohydrate, or total energy intake.

The mean (SE) cholesterol concentration for the four seven day periods is shown in fig 2 . The cholesterol concentration was significantly higher $(\mathrm{P}=0.03)$ within the first seven days in the $10 \%$ group than in the $20 \%$ group. The mean difference increased over the study period as the mean cholesterol concentration in the $10 \%$ group rose, unlike the $20 \%$ group.

A similar pattern was seen for the triglyceride concentration (fig 3). Non-esterified fatty acid concentrations were not increased in either group and there was no significant difference between the groups (data not presented). The concentration of $\beta$ hydroxy butyrate was always less than $0.35 \mathrm{mmol} / 1$.

Median weight gains during the study were $4.7 \mathrm{~g} / \mathrm{kg} /$ day and $5.3 \mathrm{~g} / \mathrm{kg} /$ day $(\mathrm{P}=0.93)$ in the $10 \%$ and $20 \%$ groups, respectively. There was also no difference in length or head circumference gain in the two groups.

The clinical outcome of the infants is shown in table 2. Retinopathy of prematurity (ROP) was significantly more common in the infants who had received $20 \%$ Lipofundin-three of these had grade 1, 13 grade 2, and 1 grade 3 -compared with 0,2 , and 3 in the $10 \%$ group. There was no significant difference in other clinical outcomes.

\section{Discussion}

This study showed that premature infants have better biochemical tolerance of $20 \% \mathrm{MCT} /$

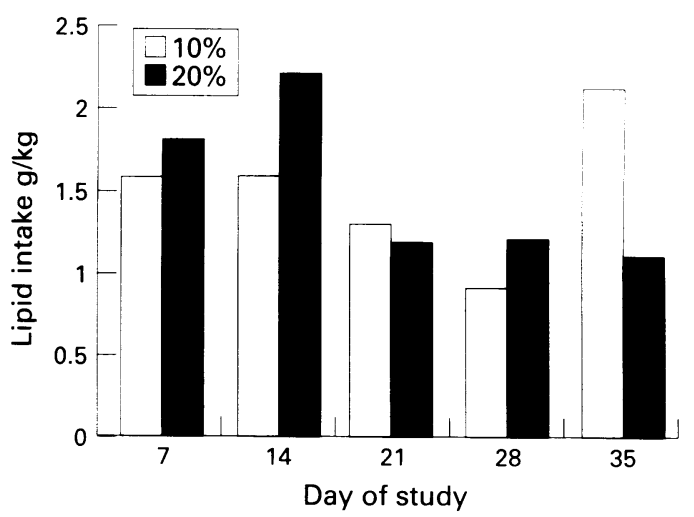

Figure 1 Median parenteral lipid intake (g/kg/day)

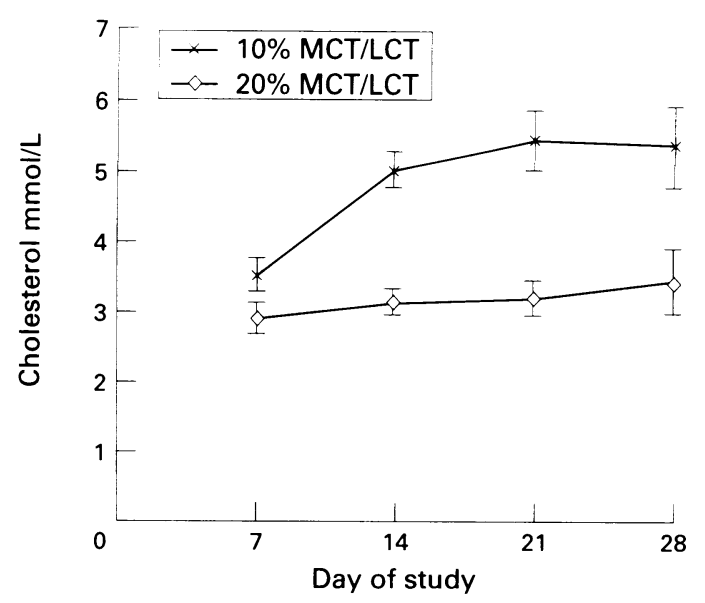

Figure 2 Mean (SEM) cholesterol concentrations in both groups 
Table 1 Patient characteristics (values are given as mean (SD))

\begin{tabular}{lcccc}
\hline & $\begin{array}{c}10 \% M C T / L C T \\
(n=38)\end{array}$ & $\begin{array}{l}20 \% M C T / L C T \\
(n=37)\end{array}$ & P value \\
\hline Birthweight (g) & $1221(607)$ & $1164(606)$ & 0.04 \\
Gestational age (weeks) & $28.9(3.2)$ & $28.2(3.2)$ & 0.87 \\
Number (\%) small for gestational age & $16(42)$ & $9(24)$ & 0.10 \\
$\begin{array}{l}\text { Number (\%) requiring intermittent } \\
\text { positive pressure ventilation }\end{array}$ & $24(63)$ & $26(70)$ & 0.10 \\
CRIB score & $4.4(3.4)$ & $6.8(3.9)$ & 0.01 \\
\hline
\end{tabular}

Table 2 Clinical outcomes of both groups

\begin{tabular}{|c|c|c|c|}
\hline & $\begin{array}{l}10 \% M C T / L C T \\
(n=38)\end{array}$ & $\begin{array}{l}20 \% M C T / L C T \\
(n=37)\end{array}$ & $P$ value \\
\hline Days of IPPV mean (SD) & $10.3(13.7)$ & $14.8(16.5)$ & 0.20 \\
\hline Days of phototherapy mean (SD) & $3.9(4.4)$ & $3.1(2.8)$ & 0.33 \\
\hline Number $(\%)$ developing BPD & $7 \quad(18)$ & 10 (27) & 0.37 \\
\hline $\begin{array}{l}\text { Number (\%) developing intraventricular } \\
\text { haemorrhage }\end{array}$ & $16(42)$ & $15(40)$ & 0.59 \\
\hline Number (\%) developing ROP & $5 \quad(13)$ & $17 \quad(46)$ & 0.001 \\
\hline
\end{tabular}

LCT emulsion than $10 \%$ emulsion. The two emulsions differ only in the amount of phospholipid emulsifier infused per gram of triglycerides (the phospholipid:triglyceride weight ratio is 0.12 for $10 \%$ and 0.06 for $20 \%$ emulsion). This produces fat emulsion particles consisting of a triglyceride core surrounded by a phospholipid coat. Once in the bloodstream apolipoproteins of the $\mathrm{A}, \mathrm{C}$, and $\mathrm{E}$ classes migrate to join the particle coat. Binding to lipoprotein lipase at the capillary endothelium then takes place, with hydrolysis releasing free fatty acids into the circulation. The amount of phospholipid emulsifier present in fat emulsions is higher than that in naturally occurring chylomicrons. The excess has been shown to exist in phospholipid bilayers resembling liposomes. ${ }^{21}$ In $10 \%$ emulsions two thirds of the phospholipids are present as liposomes, compared with one third in the $20 \%$ emulsion. This means that $10 \%$ emulsions have four times the liposomal content of $20 \%$ emulsions. In LCT emulsions these liposomes, along with the redundant outer coat of the triglyceride rich particles after hydrolysis has taken place, accumulate large amounts of endogenous cholesterol. A low density lipoprotein complex known as lipoprotein $\mathrm{X}$ is then formed. ${ }^{22}$ Lipo-

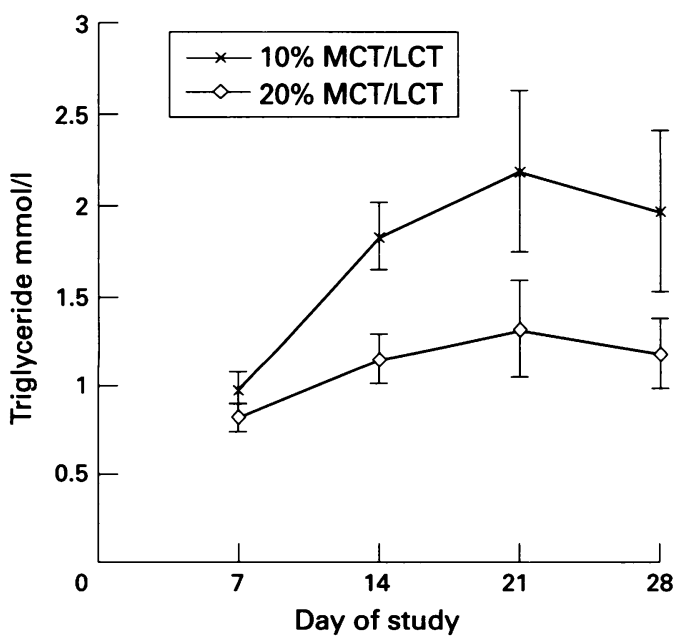

Figure 3 Mean (SEM) triglyceride concentrations in both groups protein $\mathrm{X}$ competes for lipoprotein lipase binding sites, thus increasing triglyceride concentrations. ${ }^{23}$

The biochemical results from this study show a gradual rise in cholesterol and triglyceride in the group receiving $10 \% \mathrm{MCT} / \mathrm{LCT}$ emulsion. This suggests that lipoprotein $\mathrm{X}$ is being formed in MCT/LCT emulsion use in the same way as in pure LCT emulsion. Ketone body production rises during pure MCT infusion in animal studies. ${ }^{24} \beta$ hydroxy butyrate concentrations remained low throughout the study period in both groups. The most likely explanation for this is the adequate glucose supply provided, but it is possible that increased production was matched by high utilisation by the developing brain. Non-esterified fatty acids (which have a half life of one to two minutes in the circulation) were not increased, showing that systemic oxidative removal mechanisms were not saturated at infusion rates of $3 \mathrm{~g} / \mathrm{kg} /$ day. This is important as neurological toxicity has been shown to occur with an increase in plasma medium chain fatty acids. ${ }^{25}$

The significantly higher rate of retinopathy of prematurity in the group receiving $20 \%$ emulsion was unexpected. This disorder is associated with the use of lipid emulsion. Hammerman and Aramburo ${ }^{14}$ showed that infants who received Intralipid were more likely to develop it than those having fat free parenteral nutrition. They suggested a role for either prostanoids or lipid peroxidation. But the two groups in our study received the same quantity of both medium and long chain triglycerides, differing only in the quantity of phospholipid infused. The explanation may that the $20 \%$ group were sicker at the time of randomisation, as indicated by the difference in CRIB scores. The CRIB score gives only a mortality risk and therefore cannot be used statistically to correct the risk of retinopathy of prematurity.

In conclusion, this study shows that preterm infants have better biochemical tolerance to $20 \% \mathrm{MCT} / \mathrm{LCT}$ emulsion than $10 \%$, as has been shown for pure LCT emulsion. A possible association between the use of intravenous lipids and retinopathy of prematurity requires further study.

Dr Cairns was supported by a Research fellowship from the Royal Victoria Hospital, Belfast. B Braun Ltd provided funding for sample analysis.

1 Chandra RK. Nutrition, immunity and infection. Presen knowledge and future directions. Lancet 1983;i:688-91.

2 Wilson DC, McClure BG, Dodge JA. The influence of nutrition on neonatal respiratory muscle function. Intensive Care Med 1992;18:105-8.

3 Frank L, Sosenko IRS. Undernutition as a major contributing factor in the pathogenesis of bronchopulmonary dysplasia. Am Rev Respir Dis 1988;138:725-9.

4 Leuba G, Rabinowicz T. Long-term effects of postnatal undernutrition and maternal malnutrition on mouse cerebral cortex. Exp Brain Res 1979; 37:299-308.

5 Lucas A, Morley R, Cole TJ, Gore SM, Davis JA, Bamford MFM, et al. Early diet in preterm babies and developmental status in infancy. Arch Dis Child 1989; $1: 570-8$.

6 Lee EJ, Simmer K, Gibson RA. Essential fatty acid deficiency in parenterally fed preterm infants. $\mathcal{F}$ Paediatr Child Health 1993;29:51-5.

7 Dhanireddy $R$, Hamosh $M$, Sivasubramanian $K N$, Chowdhry P, Scanlon JW, Hamosh P. Postheparin lipolytic activity and intralipid clearance in very low birth weight infants. $\mathcal{F}$ Paediatr 1981;98: 617-22. 
8 Yeh YY, Cooke RJ, Zee P. Impairment of lipid emulsion metabolism associated with carnitine insufficiency in premature infants. F Paediatr Gastoenterol Nutr 1985;4: 795-8.

9 Bellinati-Pires R, Waitzberg DL, Salgado MM, CarneiroSampaio MMS. Effect of medium and long chain triglycerides on human neutrophil migration. Brazilian $\mathcal{f} \mathrm{Med}$ Biol Res 1992; 25:369-73.

10 Park W, Paust H, Schroder H. Lipid infusion in premature infants suffering from sepsis. $\mathcal{f}$ Parent Ent Nutr 1984;290-2.

11 Brans YW, Andrew DA, Carillo DW, Dutton EP, Menchala EM, Puelo-Scheppke BA. Tolerance of fat emulsions in very low birth weight neonates. Am $¥$ Dis Child 1988;142:145-52.

12 Pereira GP, Fox WW, Stanley CA, Baker L, Schwatz JC Decreased oxygenation and hyperlipidaemia during intravenous fat infusions in premature infants. Pediatrics 1980 66:26-30.

13 Cooke RWI. Factors associated with chronic lung disease in preterm infants. Arch Dis Child 1991;66:776-9.

14 Hammerman C, Aramburo MJ. Decreased lipid intake reduces morbidity in sick premature neonates. $\mathcal{F}$ Pediatr 1988;113:1083-8.

15 Johnson RC, Cotter R. Metabolism of medium chain triglygeride lipid emulsion. Nutr Int 1986;2:150-8.

16 Rubin M, Harell D,Naor N, Mosher A, Wielunsky E, Merlob $\mathrm{P}$, et al. Lipid infusion with different triglyceride cores (long chain vs medium chain/long chain triglycerides): effect on plasma lipids and bilirubin binding in premature Nut 1991;15:642-6.

17 Lima LAM, Murphy JF, Stansbie D, Rowlandson P, Gray OP. Neonatal parenteral nutrition with a fat emulsion containing medium chain triglycerides. Acta Paediat Scand 1988;77:332-9.

18 Haumont D, Deckelbaum RJ, Richelle $M$, Dahlan $W$, Coussaert, Bihain BE, et al. Plasma lipid and plasma lipoprotein concentrations in low birth weight infants given parenteral nutrition with twenty or ten percent lipid emulsion. $\mathcal{F}$ Pediatr 1989;115:787-93.

19 Halliday HL,McClure BG, Reid McC. Handbook of neonatal intensive care. 3rd edn. London: Ballière Tindall, 1989.

20 The International Neonatal Network. The CRIB (clinical risk index for babies) score: a tool for assessing initial neonatal risk and comparing performance of neonata intensive care units. Lancet 1993;342:193-8.

21 Groves MJ, Wineberg M, Brain APR. The presence of liposomal material in phosphatide stabilized emulsions. $₹ D$ ispers Sci Technol 1985;6:237-42.

22 Griffin E, Brekenridge WC, Kuksis A, Bryan MN, Angel A Appearance and characterization of lipoprotein $\mathrm{X}$ durin continuous Intralipid infusions in the neonate. $\mathcal{F}$ Clin Inves 1979;64:1703-12.

23 Seidel D. Lipoproteins in liver disease. 7 Clin Chem Clin Biochem 1987;25:541-51.

24 Cotter R, Taylor CA, Jonson R, Rowie WB. A metabolic comparison of a pure long chain triglyceride lipid (MCT)-LCT combination emulsions in dogs. Am $¥$ Clin Nutr 1987;45:927-39.

25 Miles JM, Cattalini M, Sharbrough FW, Wold LE, Wharen $\mathrm{RE}$, Gerich JE, et al. Metabolic and neurologic effects of an intravenous medium chain triglyceride emulsion. $f$ Paren Ent Nutr 1991;15:37-41. 